\title{
Mass Spectrometric Lipid Profiles of Picosecond Infrared Laser-Generated Tissue Aerosols Discriminate Different Brain Tissues
}

\author{
Marcus Wurlitzer, (D) ${ }^{1}$ Elisabeth Hessling, ${ }^{1}$ Karsten Rinas, ${ }^{1}$ MarcelineManka Fuh, ${ }^{1}$ Hannes Petersen, ${ }^{2}$ \\ Franz Ricklefs, ${ }^{3}$ Katrin Lamszus, ${ }^{3}$ Jan Regelsberger, ${ }^{3}$ Stephanie Maier, ${ }^{4}$ Sebastian Kruber, ${ }^{4}$ \\ Nils-Owe Hansen, ${ }^{4}$ RJDwayne Miller, ${ }^{4,5}$ and Hartmut Schlüter ${ }^{1 *}$ \\ ${ }^{1}$ Department of Mass Spectrometric Proteomics, Institute for Clinical Chemistry and Laboratory Medicine, University \\ Medical Center Hamburg-Eppendorf, Martinistr. 52, 20246 Hamburg, Germany \\ ${ }^{2}$ Department of Otorhinolaryngology, Head and Neck Surgery and Oncology, University Medical Center \\ Hamburg-Eppendorf, Martinistraße 52, 20246 Hamburg, Germany \\ ${ }^{3}$ Department of Neurosurgery, University Medical Center Hamburg-Eppendorf, Martinistraße 52, 20246 Hamburg, \\ Germany \\ ${ }^{4}$ Atomically Resolved Dynamics Division, Max Planck Institute for the Structure and Dynamics of Matter, Luruper \\ Chaussee 149, 22761 Hamburg, Germany \\ ${ }^{5}$ Departments of Chemistry and Physics, Lash Miller Chemical Laboratories, University of Toronto, 80 St. George Street, \\ LM245A, Toronto, Ontario, M5S 3H6, Canada
}

Background and Objectives: A picosecond infrared laser (PIRL) has recently been demonstrated to cut biological tissue without scar formation based on the minimal destructive action on the surrounding cells. During cutting with PIRL, the irradiated tissue is ablated by a cold vaporization process termed desorption by impulsive vibrational excitation. In the resulting aerosol, all molecules are dissolved in small droplets and even labile biomolecules like proteins remain intact after ablation. It is hypothesized that these properties enable the PIRL in combination with mass spectrometry as an intelligent laser scalpel for guided surgery. In this study, it was tested if PIRL-generated tissue aerosols are applicable for direct analysis with mass spectrometry, and if the acquired mass spectra can be used to discriminate different brain areas.

Materials and Methods: Brain tissues were irradiated with PIRL. The aerosols were collected and directly infused into a mass spectrometer via electrospray ionization without any sample preparation or lipid extraction.

Results: The laser produced clear cuts with no marks of burning. Lipids from five different classes were identified in the mass spectra of all samples. By principal component analysis the different brain areas were clearly distinguishable from each other.

Conclusions: The results demonstrate the potential for real-time analysis of lipids with a PIRL-based laser scalpel, coupled to a mass spectrometer, for the discrimination of tissues during surgeries. Lasers Surg. Med. (C) 2019 Wiley Periodicals, Inc.

Key words: picosecond infrared laser; laser ablation; mass spectrometry; lipid analysis; brain lipids; diagnostic tools; brain surgery; PIRL; DIVE

\section{INTRODUCTION}

The classification of biological samples is an important step in the treatment of malignant diseases. Current methods to verify the presence of tumorous tissue from biopsies or surgical resections rely on histology or immunohistochemistry. Intraoperative frozen section histopathology, which is used during surgery to give the surgeon feedback on the tumor content of the resected tissue, takes roughly 30 minutes and is usually limited to a few specimens. Their pathological assessment provides a general statement about the presence of tumor cells, but the extent or the exact placement of the tumor within the operative field cannot be determined from histopathology alone. A real-time classification technique which provides fast, accurate, minimally-invasive tissue diagnostics in situ is therefore highly desirable [1]

To overcome the limitations of tumor assessment in cancer surgery, several intraoperative methods based on the tissue identification by mass spectrometry are being 
developed [2-5]. These methods are all based on the mass spectrometric analysis of lipids and metabolites, but use different desorption techniques to sample the tissues, including an electrosurgical knife [2], desorption electrospray ionization mass spectrometry (DESI-MS) [4], resonant infrared laser ablation (RIR-LA) [3], and dissolution of molecules by small water droplets [5].

In this study, a picosecond infrared laser (PIRL) based on the desorption by impulsive vibrational excitation (DIVE) is examined as another method to sample biological tissues for subsequent mass spectrometric identification. The DIVE ablation is a laser technique which has shown the potential for significantly less thermal damage to biological tissue compared with electrosurgery or conventional laser surgery through a more focused energy deposition by pulse durations in the picosecond time scale [6]. Targeting the $\mathrm{O}-\mathrm{H}$ stretch vibration's absorption band in water at $3 \mu \mathrm{m}$ wavelength and with pulse durations of $10-400 \mathrm{ps}$, no significant amount of energy is transferred to the surrounding tissue, neither in thermal form nor by acoustic shockwaves or by ionizing radiation, which would lead to degradation of biomolecules. Instead, the absorbed energy is efficiently coupled to translation motion and accelerates the ablation process instead of being lost to thermal or acoustic transients outside the excited volume [7]. Thus, irradiation with PIRL minimizes the damage to the surrounding tissue and generates an aerosol with all molecules from the tissue solubilized in small droplets.

Recent studies have demonstrated that surgical incisions of skin and laryngeal tissues, including vocal folds, made with the PIRL lead to heavily reduced collateral damage [8-10]. Labile biomolecules, such as proteins, remain in their original chemical composition, and even enzymatic activities are maintained [11,12]. In situ application of the PIRL has been demonstrated in a wound healing study, where surgical incisions made with the PIRL on the skins of rats showed a significant reduction in scar-formation [10]. Due to the soft properties of the DIVE ablation process, PIRL-based sample probing is particularly interesting for applications where minimal tissue damage is desired, like brain surgery. The PIRL laser produces clear cuts while reducing scar formation and thermal damage to the remaining tissue and releases intact biomolecules into the aerosol. Therefore, the PIRL is an ideal tool for soft in situ sampling of tissues for mass spectrometric analysis. In this study, it is tested if different types of tissues can be distinguished by the mass spectrometric lipid profiles of their PIRL ablation products.

\section{MATERIALS AND METHODS}

\section{Tissue Preparation}

The brain of a 3-year-old mini pig (Sus scrofa domesticus) was dissected and six different anatomical regions, including cerebral cortex, cerebellar cortex, corpus callosum, thalamus, mesencephalon, and hypophysis, were prepared by a neurosurgeon. The areas were cut into pieces of
$1 \times 1 \times 0.5 \mathrm{~cm}$ and snap-frozen on a piece of cork in liquid nitrogen. Tissues were stored at $-80^{\circ} \mathrm{C}$ until usage.

\section{DIVE Ablation With the PIRL Laser}

A prototype laser system (PIRL-HP2-1064 OPA-3000; Attodyne Inc., Toronto, Canada) was used for the ablation of the tissue. The laser beam emitted light at a wavelength of $3 \mu \mathrm{m}$ with a pulse duration of $400 \mathrm{ps}$, a pulse energy of $700 \mu \mathrm{J}$, at a repetition rate of $1,000 \mathrm{~Hz}$. A custom-made beam guiding and auto-focus system with a scanning mirror before the final focusing lens was set up to direct the laser beam evenly in an overall area of $5 \times 5 \mathrm{~mm}$ [2]. The laser beam had to be focused on the tissue surface with a tolerance of approximately $1 \mathrm{~mm}$ to ensure optimal ablation conditions and prevent thermal damage to the tissue. The optical auto-focus system provided a fast and accurate focusing by measuring the distance to the sample surface with a camera and adjusting the position of the focusing lens in real time.

The tissue was placed in an ablation chamber on a precooled copper block to optimize the capturing of the ablation plume and keep the tissue frozen during the ablation process. The tissue was irradiated with the laser for 4 minutes. The plume was transferred via polytetrafluoroethylene (PTFE) tubing into a falcon tube by a surgical suction pump. Cooling of the falcon tube with liquid nitrogen led to immediate condensation and freezing of the plume at the wall of the tube. The condensed ablation plumes were stored at $-20^{\circ} \mathrm{C}$ until the mass spectrometric experiment.

\section{Mass Spectrometry}

Mass spectrometry was performed on an Orbitrap Q Exactive (Thermo Fisher Scientific, Waltham, MA) mass spectrometer with a nano-ESI source. Eight microliters of each sample were applied via flow injection with an Agilent LC 1200 Series (Agilent Technologies, Santa Clara, CA) at a flow rate of $0.5 \mu \mathrm{l} / \mathrm{min}$. The flow injection setup allowed for a constant signal of each sample of up to 15 minutes. Spectra were acquired in positive and negative full-scan mode in an $\mathrm{m} / z$ range from 400 to 1,500 at a resolution of 140,000 . The maximum injection time was set to 2.0 seconds to maximize the spectrum quality.

\section{Data Analysis}

For each sample, a five-spectra average was used for statistical analysis. The spectra were exported as peak lists with Xcalibur 2.1 (Thermo Fisher Scientific) to a comma-separated table. Further processing was performed with Mathematica 10.0 (Wolfram Research). The peak lists of all spectra were imported, and signals were matched among the samples with a tolerance of $5 \mathrm{ppm}$. The signal intensities were normalized sample-wise to the median. Signals that occurred in three or more samples were included for the principal component analysis (PCA).

The LIPID MAPS Structure Database (LMSD) was used for identification of the lipids. The search was performed in all lipid categories with a mass tolerance of 
$m / z \pm 0.005$ and ion species $[\mathrm{M}-\mathrm{H}]^{-}$and $[\mathrm{M}-2 \mathrm{H}]^{2-}$. Comparison of the observed isotopic pattern of a specific signal with its theoretical isotopic pattern, calculated with ChemCalc (http://www.chemcalc.org/main), allowed for discrimination between multiple candidates of different ion species. The search output was reduced to the total number of $\mathrm{C}$ atoms and double bonds in the variable fatty acid chains.

\section{RESULTS}

Six different areas from a pig brain including cerebral cortex, cerebellar cortex, corpus callosum, thalamus, mesencephalon, and hypophysis were subjected to PIRL ablation. An area of $5 \times 5 \mathrm{~mm}$ [2] was ablated from three separately prepared tissue blocks per brain area (two for cerebellar cortex). In 4 minutes ablation time, approximately $1 \mathrm{ml}$ of aerosol condensate per sample was collected in the cooling trap (Fig. 1).

The soft ablation of the laser produced a square-sized hole with smooth borders in the ablated tissues. No marks of burning were visible on the tissue pieces (Fig. 2). The condensates of the tissue aerosols were directly infused into the nano-ESI source of a mass spectrometer by flow injection and analyzed in negative ion mode. Characteristic mass spectra of two different brain areas are shown in Figure 3.
Average spectra from each tissue block were converted to peak lists and subjected to a PCA. The six different brain areas were clearly separated by the first two components of the PCA (Fig. 4). For each cluster of samples from one brain area, the ellipse is drawn around the mean with radii of the doubled standard deviation along each principal component. All brain areas are separated by at least the doubled standard deviations in terms of confidence in identifying the different tissue types, except for a small overlap between thalamus and cerebellar cortex.

To identify the $m / z$ signals with the highest contribution to the separation of the brain areas, the total weight in the first three principal components was calculated for each signal as the Euclidean distance from 0, resulting in a number between 0 (no weight) and 1 (maximum weight), as listed in Table 1.

\section{Identification of Lipid Signals}

The $\mathrm{m} / z$ values of the monoisotopic signals were searched against the LIPID MAPS Structure Database (LMSD). A mass deviation of $0.005 \mathrm{~m} / \mathrm{z}$ from the calculated mass was allowed and the isotopic pattern had to be consistent with the ion species. Table 1 summarizes the closest match for each lipid signal in the LIPID MAPS database. Due to the existence of multiple isomers

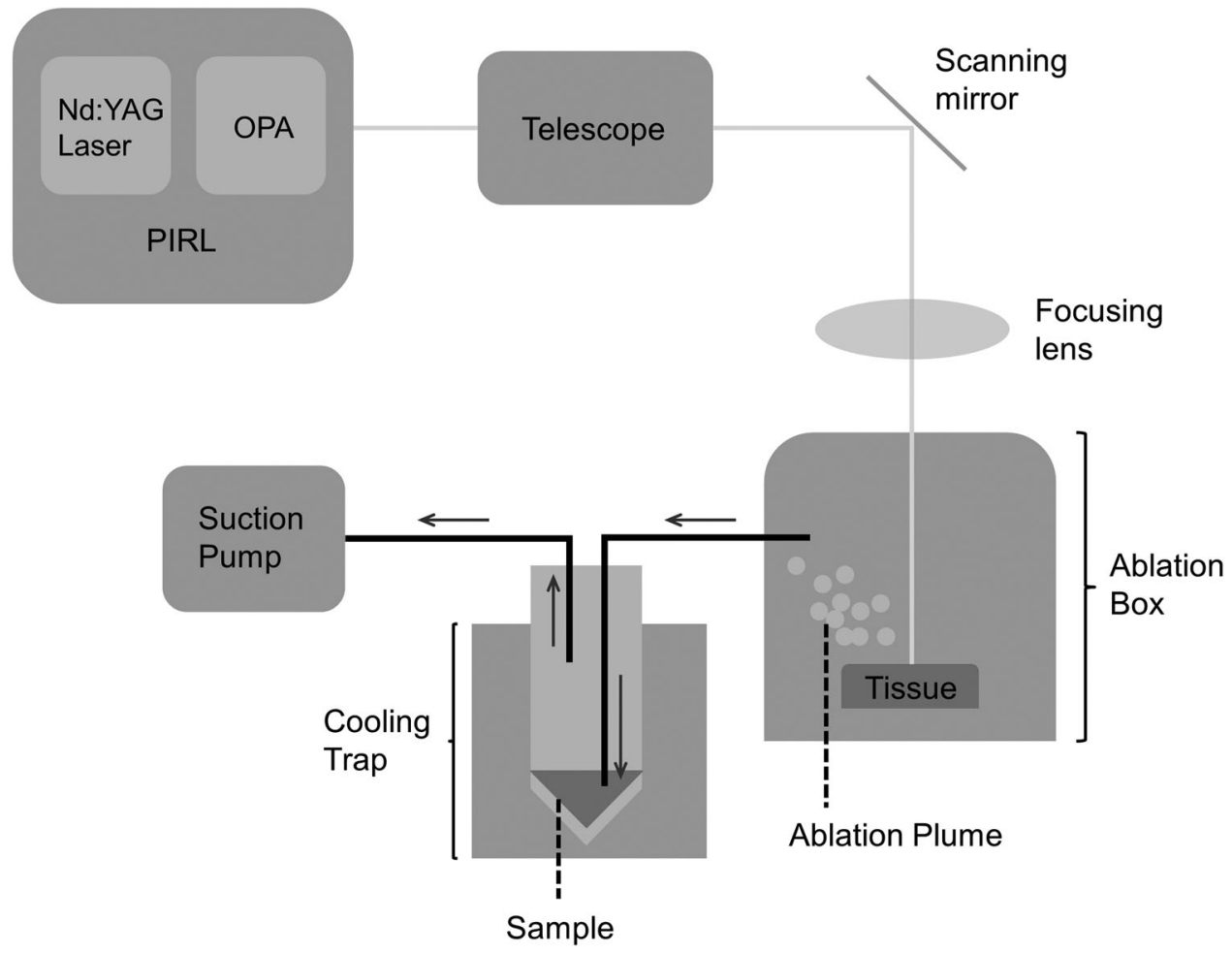

Fig. 1. Experimental setup of the laser ablation and plume collection. The laser beam is transferred through a custom-made beam guiding and auto-focus system with a scanning mirror, which allows for fast and precise movement of the laser spot on the tissue surface. It passes through a sapphire window into the closed ablation box. The tissue aerosol is sucked into a polytetrafluoroethylene tubing by a suction pump and condensed in a cooling trap filled with liquid nitrogen. 


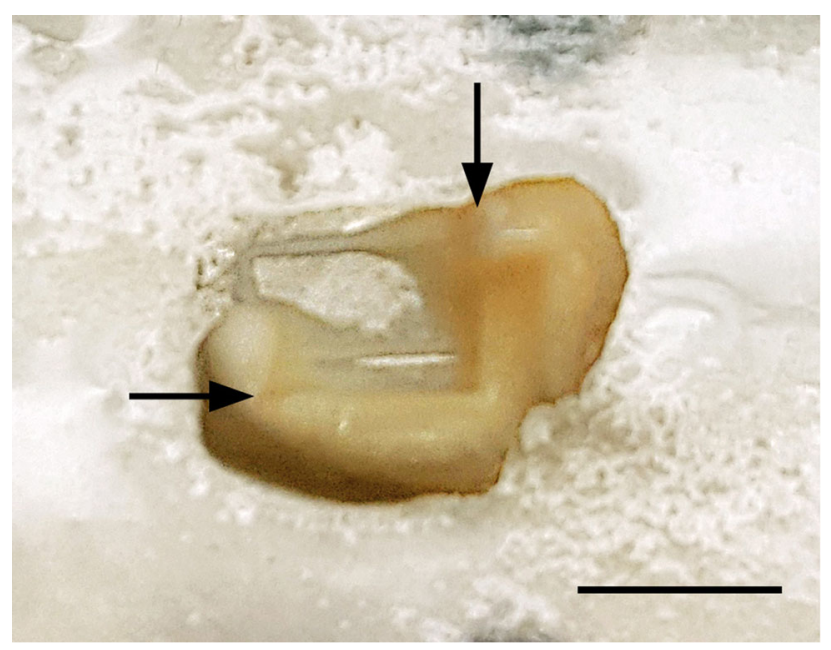

Fig. 2. Photograph of a piece of brain tissue after laser ablation. Arrows indicate the edges of the ablated area $\left(5 \times 5 \mathrm{~mm}^{2}\right)$. The upper left part of the tissue was ablated completely. The picosecond infrared laser produced a smooth cut without any visible burning marks. Scale bar $=5 \mathrm{~mm}$ [Color figure can be viewed at wileyonlinelibrary.com].

sharing the same monoisotopic mass, only the sum formula is given.

Signals were identified as phosphatidylserines (PS), phosphatidylinositols (PI), a phosphatidic acid (PA), sulfatides (ST), as well as hydroxylated sulfatides (ST$\mathrm{OH})$. One phosphatidylserine, $\mathrm{PS}(40: 6)$, was detected as a singly and a doubly charged ion species.

\section{DISCUSSION}

In this study, we demonstrated that tissue ablation with PIRL allows for a direct mass spectrometric analysis without any further sample preparation. The majority of the detected signals in the brain tissue samples were identified as lipid species, including PS, PI, PAs, ST, and
ST-OH. No collateral damage to the brain tissue adjacent to the ablated area was visible after using the PIRL for tissue sampling. Moreover, our wound healing studies have shown that the removal of tissue material with PIRL leads to effectively scar-free healing [10].

The PIRL is an experimental setup in an optical lab and is under active development to optimize ablation results and usability. A newly developed auto-focus system improved handling and accuracy of the ablation procedure compared with earlier studies with manual focus. Automatic focusing can be considered a requirement for any practical use of the laser in an operating theater. It can be achieved either by a suitable auto-focus system, as in our study, or by guiding the laser through a fiber, which is held by the hand of the surgeon and brought into direct contact with the tissue $[13,14]$.

The current sampling setup including the ablation chamber, PTFE suction, and the cooling trap has been used for the analysis of proteins in earlier studies with the PIRL laser. Condensation and freezing of the ablation plume allows for sample preparation procedures such as tryptic digestion, which is essential for the mass spectrometric analysis of proteins. In contrast to protein analysis, the high abundance and the good desolvation and ionization efficiency of lipids result in intense mass spectrometric signals even without specific preparation of the samples. We were able to skip the sample preparation procedure entirely and analyze and characterize the brain samples directly from the condensed ablation plumes. We therefore conclude that the ablation plume can be transferred directly into the source of a mass spectrometer to generate signals from lipids, making the PIRLMS method suitable for on-line classification of biological tissues.

A long ablation and collection time of 4 minutes was chosen to ensure the collection of sufficient sample volumes despite potential losses in the tube transfer process. The average collection volume per sample was
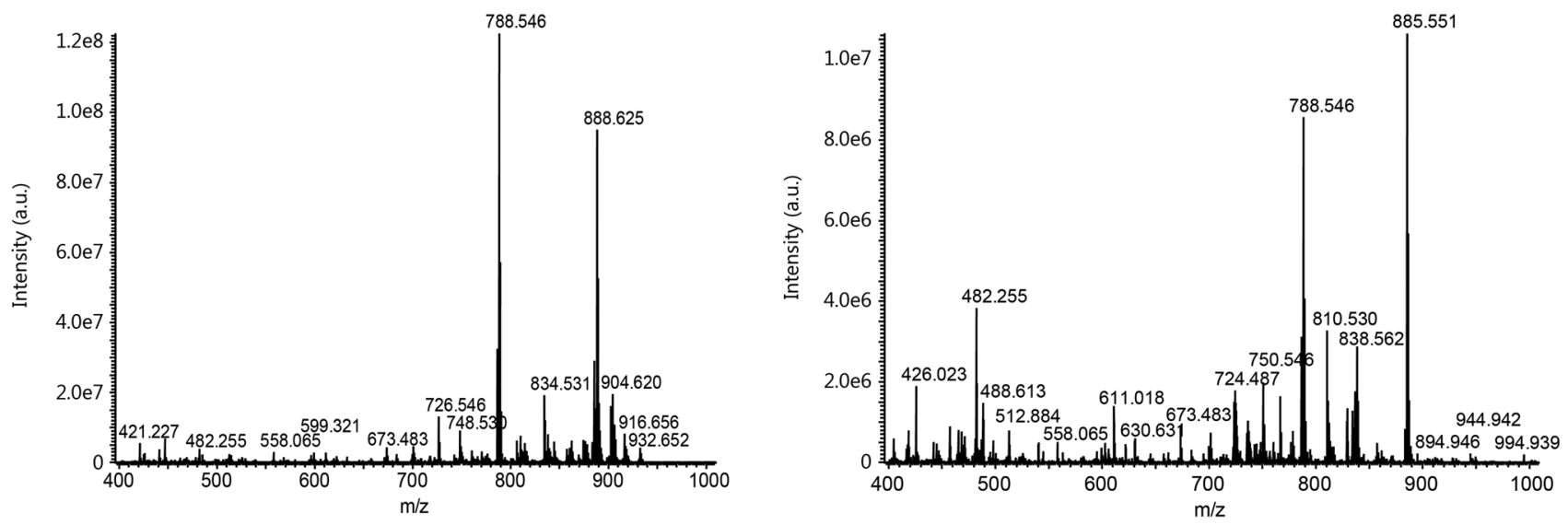

Fig. 3. Electrospray ionization-mass spectrometry full-scan spectra acquired from the corpus callosum (left) and the hypophysis (right) of the pig brain in the negative ion mode. Most signals were detected in an $\mathrm{m} / \mathrm{z}$ range of $700-900$, representing the $\mathrm{m} / \mathrm{z}$ range of most singly charged lipids. Doubly charged species were found in an $\mathrm{m} / z$ range of $400-550$. 


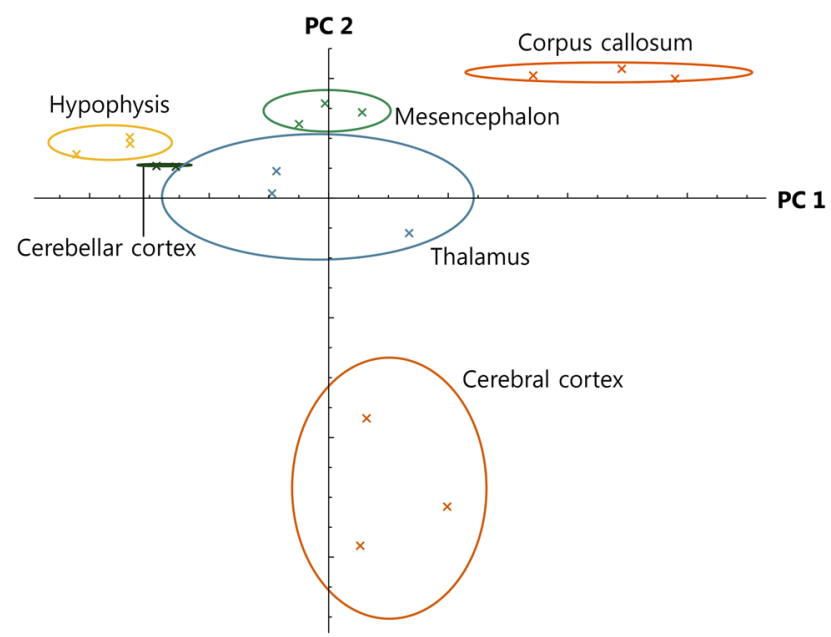

Fig. 4. Principal component analysis (PCA) plot of brain area mass spectra. The first two components of the PCA are shown. Each dot represents one sample, each color represents one brain area. The circles indicate the doubled standard deviation of the samples of each brain area.

approximately $1 \mathrm{ml}$. Since only $8 \mu \mathrm{l}$ were needed for mass spectrometric analysis and no further sample processing was involved, the ablation time can potentially be reduced to a few seconds. The samples were kept frozen on a precooled copper block to minimize degradation of the previously excised tissue material. Previous studies have shown that the soft properties of the PIRL ablation do not change when fresh tissue is irradiated at room temperature, so cooling of the tissue is not necessary when the laser is used intraoperatively [10].

The lipid profiles of the different brain areas were separated in a principal component analysis (PCA). Although the sample size was small ( $n=3$ for each group, with $n=2$ in the cerebellar cortex group), it is notable that the brain areas formed distinct clusters in the PCA. Being an unsupervised transformation of the data, the PCA is not specifically trained to classify known groups of samples, but rather separates the data points by a linear combination of the input variables (lipid signal intensities) to account for the highest possible variance. This suggests that the differences in the lipid composition of different brain areas are indeed the greatest source of variability in the data set, rather than statistical noise or artifacts from the experimental setup, such as variances in laser ablation or collection losses. Although the tissue samples were separated by at least the doubled standard deviation, except for thalamus and cerebellar cortex, the discriminative power of the PCA is limited by the small number of samples. Also, a supervised classification method, like a support vector machine, can potentially classify the samples with higher accuracy, but is likely to be overfitted with small sample sizes as in this study, and therefore was not used. Subsequent studies with a larger number of samples are necessary to fully explore the capacity of the PIRL-MS method to distinguish tissue samples.

Lipids are the basic constituents of the brain, providing about $60 \%$ of its dry weight [15]. The macroscopic appearance of different brain areas is mainly determined by its amount of myelin, which is rich in lipids and appears white. The brain cortex contains low amounts of myelin and appears as gray matter. In contrast, the corpus callosum consists of white matter fibers connecting both hemispheres and is rich in myelination [16]. This differentiation gets more difficult with inhomogeneous regions like the thalamus, which consists mostly, but not only, of gray matter. The mesencephalon hosts many different areas and functions and consists of a mixture of white and gray matter. When relating the separation observed in the PCA (Fig. 4) to the biology of the brain areas, the principal component 2 (PC2) axis coincides with the amount of myelination (gray vs. white matter). The cerebral cortex lies on the bottom end of the axis

TABLE 1. List of Monoisotopic Signals With the Closest Matching Lipid Species in the LIPID MAPS Database

\begin{tabular}{lccccc}
\hline$m / z$ (obs.) & Ion species & Sum formula & Lipid & $\Delta m / z$ (ppm) & PC 1-3 weight \\
\hline 834.531 & {$[\mathrm{M}-\mathrm{H}]^{-}$} & $\mathrm{C}_{46} \mathrm{H}_{77} \mathrm{O}_{10} \mathrm{PN}$ & $\mathrm{PS}(40: 6)$ & 2.40 & 0.81 \\
788.547 & {$[\mathrm{M}-\mathrm{H}]^{-}$} & $\mathrm{C}_{42} \mathrm{H}_{79} \mathrm{O}_{10} \mathrm{PN}$ & $\mathrm{PS}(36: 1)$ & 2.28 & 0.76 \\
888.626 & {$[\mathrm{M}-\mathrm{H}]^{-}$} & $\mathrm{C}_{48} \mathrm{H}_{90} \mathrm{O}_{11} \mathrm{NS}$ & $\mathrm{ST}(24: 1)$ & 1.91 & 0.67 \\
885.552 & {$[\mathrm{M}-\mathrm{H}]^{-}$} & $\mathrm{C}_{47} \mathrm{H}_{82} \mathrm{O}_{13} \mathrm{P}$ & $\mathrm{PI}(38: 4)$ & 2.15 & 0.48 \\
512.884 & {$[\mathrm{M}-2 \mathrm{H}]^{2-}$} & $\mathrm{C}_{58} \mathrm{H}_{108} \mathrm{O}_{11} \mathrm{PN}$ & $\mathrm{PS}-\mathrm{NAc}(52: 1)$ & 2.36 & 0.22 \\
838.562 & {$[\mathrm{M}-\mathrm{H}]^{-}$} & $\mathrm{C}_{46} \mathrm{H}_{81} \mathrm{O}_{10} \mathrm{PN}$ & $\mathrm{PS}(40: 4)$ & 1.44 & 0.20 \\
416.762 & {$[\mathrm{M}-2 \mathrm{H}]^{2-}$} & $\mathrm{C}_{46} \mathrm{H}_{76} \mathrm{O}_{10} \mathrm{PN}$ & $\mathrm{PS}(40: 6)$ & 2.29 & 0.19 \\
786.531 & {$[\mathrm{M}-\mathrm{H}]^{-}$} & $\mathrm{C}_{42} \mathrm{H}_{77} \mathrm{O}_{10} \mathrm{PN}$ & $\mathrm{PS}(36: 2)$ & 2.33 & 0.13 \\
904.620 & {$[\mathrm{M}-\mathrm{H}]^{-}$} & $\mathrm{C}_{48} \mathrm{H}_{90} \mathrm{O}_{12} \mathrm{NS}$ & $\mathrm{ST}-\mathrm{OH}(24: 1)$ & 1.35 & 0.12 \\
701.515 & {$[\mathrm{M}-\mathrm{H}]^{-}$} & $\mathrm{C}_{39} \mathrm{H}_{74} \mathrm{O}_{8} \mathrm{P}$ & $\mathrm{PA}(36: 1)$ & $\mathrm{ST}(24: 0)$ &
\end{tabular}

PA, phosphatidic acid; PI, phosphatidylinositol; PS, phosphatidylserine; PS-NAc, $N$-acyl phosphatidylserine; ST, sulfatide, ST-OH, hydroxylated sulfatide.

Lipid names are given by the lipid subclass and the total number of $\mathrm{C}$ atoms and double bonds in the variable fatty acid chains. The sum formula represents the chemical composition of the detected ion species. The deviation of the observed mass-to-charge ratio $(m / z$ [obs.]) from the theoretical one is given in ppm, as well as the total weight in the first three components of the principal component analysis as the distance to zero (PC $1-3$ weight). 
(low myelination), the corpus callosum at the top end (high myelination), and mesencephalon and thalamus inbetween. The hypophysis has a special status. It consists of two lobes, the anterior lobe (anterior pituitary), which is an endocrine gland and accounts for roughly $80 \%$ of the pituitary gland, and the posterior lobe (posterior pituitary), which consists mainly of unmyelinated axons. The special character of the pituitary gland is also reflected in the PCA, where the hypophysis samples gather at the topleft hand corner of the PCA plot.

Correlations between specific lipids and brain regions can be examined with the help of the PCA loadings plot (Supplemental Fig. S1). Combined with the previous observation that the PC2 axis corresponds to the presence of myelination, the lipid signal at $\mathrm{m} / \mathrm{z}$ 834.531, which represents PS(40:6) and had the highest negative coefficient in the PC2, was an indicator for low amounts of myelination and thus for gray matter. Conversely, the signals at $\mathrm{m} / \mathrm{z} 788.547(\mathrm{PS}(36: 1)$ ) and $\mathrm{m} / \mathrm{z} 888.626$ (ST(24:1)), both with positive coefficients in the PC2, related to the presence of white matter. The PS(36:1) signal was also the strongest discriminator along the PC1 axis, separating the samples of hypophysis, where the signal intensity of this lipid was low, mesencephalon, and corpus callosum, where the highest signal intensity was detected for this lipid. The second most important discriminator in the $\mathrm{PC} 1$ was $\mathrm{m} / z$ 885.552, representing PI(38:4), where high signal intensities were detected in the hypophysis samples and low intensities in the corpus callosum samples. The same discriminative properties of the $\mathrm{m} / z 834$ and $\mathrm{m} / \mathrm{z} 888$ signals have been observed in a previous DESI-MS study of brain tissue [17]. There, $\mathrm{m} / \mathrm{z}$ 788 and $\mathrm{m} / z 885$ were also present as abundant signals, but only $\mathrm{m} / \mathrm{z} 885$ showed some discriminative power among the samples in the PCA. Notably, only gray matter, white matter, and glioma samples were compared in that study. Samples from the hypophysis or the corpus callosum were not explicitly included, which may explain why $\mathrm{m} / \mathrm{z} 788$ was not found among the discriminative signals. Considering the most dominant lipids, the results from our PIRL-MS study are consistent with the DESIMS study by Cooks et al., which is promising in terms of reliability of the methods even when different desorption techniques are used.

The surgical treatment of brain cancer is particularly challenging as the patient's prognosis is heavily determined by the accuracy of tumor removal. In glioblastoma patients, only if over $98 \%$ of tumorous tissue is removed a significant increase in survival rate is observed [18], which is as low as $20 \%$ on average for the next 5 years [19]. However, the precise intraoperative assessment of tumor margins is difficult. A spacious resection with complete elimination of the entire tumor tissue is generally impracticable due to the infiltrative growth of the tumor cells and the risk of neurological deficits resulting from resection of healthy brain tissue. Standard microsurgical approaches usually involve conventional scalpels and medical lasers. Intraoperative magnetic resonance imaging $[20]$ and fluorescence-guided surgery with 5 -aminolevulinic acid increased the number of patients with radiologically visible complete tumor resection [21-23], but both methods leave room for improvement in tumor detection [24].

Several promising approaches in the field of on-line intraoperative discrimination of tissues are currently being developed or tested in the operating room. In 2013, Balog and Takats et al. introduced an electrosurgical knife connected to a mass spectrometer for the on-line identification of tissue, named iKnife. The molecules released by the electrosurgical dissection are directly transferred into a mass spectrometer [2]. In 2014, Santagata and Agar et al. demonstrated the use of DESI-MS for intraoperative identification of brain tumors. The method was applied to tissue biopsies in the operating room, a direct application to the tissue in situ was not shown [4]. In 2016, Jarmusch and Cooks et al. used DESI-MS in positive and negative mode to distinguish brain samples of gray matter, white matter, and glioma by their lipid profiles [17]. In 2016, Fatou and Fournier et al. developed an instrument based on RIR-LA for the intraoperative classification of tissues, named SpiderMass. Tissues are ablated by an infrared laser and the aerosol is directed to a mass spectrometer for realtime analysis [3]. In 2017, a handheld sampling device named the MasSpec Pen was introduced by Zhang and Eberlin et al. Molecules from the tissue are dissolved in a small water droplet which is then sucked into the source of a mass spectrometer [5].

All of these methods use mass spectrometry to acquire spectra from desorbed tissues and discriminate tissues by one or more features found in the spectra. The iKnife and SpiderMass methods use negative ion mode signals in the mass range of $m / z 600-900$ and $m / z 200-1,000$, respectively, to discriminate the samples. These signals mainly represent lipids of various species [2,3]. With the DESI-MS method, signals in the mass range of $\mathrm{m} / \mathrm{z}$ 100-1,000 were recorded, including lipids and other metabolites [4].

While the analytical part of the methods is similar, the desorption mechanisms are greatly different. Because of the different side-effects of these desorption mechanisms, one of the methods can be favorable to the others for a particular task in surgery. The electrosurgical knife used by the iKnife involves immediate coagulation to stop bleeding during cutting. It induces, by nature, significant thermal damage to the surrounding tissue, which may limit its use for operations on sensitive tissues such as the brain. DESI has been applied to tissue sections [25] and smears [26], but not for in situ sampling of potential tumorous tissue before excision. The infrared-laser desorption used by SpiderMass comes closest to the properties of the PIRL used in our study. Both lasers excite the $\mathrm{O}-\mathrm{H}$ stretch vibration at a wavelength of $2.94 \mu \mathrm{m}$. However, the PIRL laser emits 1,000 very short pulses $(400 \mathrm{ps})$ per second with $700 \mu \mathrm{J}$ per pulse, while the SpiderMass infrared laser emits only 10 pulses per second pulse with energies between 7 and $9 \mathrm{~mJ}$. The pulse energy is higher by a factor of 10 , resulting in higher peak energy levels which are applied to the tissue. A comparative study of 
the SpiderMass and the DIVE ablation on the outcome of brain surgery would be interesting to compare the thermal side-effects of cutting brain tissues in situ.

\section{CONCLUSION}

We have demonstrated the PIRL-MS combination as an ultra-soft in situ classification method for biological tissues. The clear cuts produced by the PIRL highlight the minimal-invasive properties of the laser. The identification of lipids and the differentiation between various brain areas demonstrate that the aerosol generated by PIRL can be used for mass spectrometric tissue analysis. When used as an on-line method during surgery, the PIRL-MS combination can give molecular-level feedback on specific regions of the brain and other tissue for surgical guidance. Prospectively, the combination of minimally-invasive laser surgery and mass spectrometric guidance should lead to superior results in the efficacy of cancer removal and to maximal preservation of the surrounding healthy tissue.

\section{ACKNOWLEDGMENTS}

This work is part of the ERC Advanced Grant (ERC2011-AdG) "SUREPIRL" (GA\# 291630), and part of DIVE SPOT project (MPG-FhG cooperation project; M.FE.A.SDMA0002; Max-Planck Gesellschaft).

\section{REFERENCES}

1. Ifa DR, Eberlin LS. Ambient ionization mass spectrometry for cancer diagnosis and surgical margin evaluation. Clin Chem 2016;62(1):111-123.

2. Balog J, Sasi-Szabo L, Kinross J, et al. Intraoperative tissue identification using rapid evaporative ionization mass spectrometry. Sci Transl Med 2013;5(194):194ra93.

3. Fatou B, Saudemont P, Leblanc E, et al. In vivo real-time mass spectrometry for guided surgery application. Sci Rep 2016;6: 25919.

4. Santagata S, Eberlin LS, Norton I, et al. Intraoperative mass spectrometry mapping of an onco-metabolite to guide brain tumor surgery. Proc Natl Acad Sci U S A 2014;111(30):1112111126.

5. Zhang J, Rector J, Lin JQ, et al. Nondestructive tissue analysis for ex vivo and in vivo cancer diagnosis using a handheld mass spectrometry system. Sci Transl Med 2017;9(406):eaan3968.

6. Amini-Nik S, Kraemer D, Cowan ML, et al. Ultrafast mid-IR laser scalpel: protein signals of the fundamental limits to minimally invasive surgery. PLOS One 2010;5(9):e13053.

7. Franjic K, Miller D. Vibrationally excited ultrafast thermodynamic phase transitions at the water/air interface. Phys Chem Chem Phys 2010;12(20):5225-5239.

8. Hess M, Hildebrandt MD, Muller F, et al. Picosecond infrared laser (PIRL): An ideal phonomicrosurgical laser? Eur Arch Otorhinolaryngol 2013;270(11):2927-2937.

9. Bottcher A, Kucher S, Knecht R, et al. Reduction of thermocoagulative injury via use of a picosecond infrared laser (PIRL) in laryngeal tissues. Eur Arch Otorhinolaryngol 2015;272(4):941-948.
10. Petersen H, Tavakoli F, Kruber S, et al. Comparative study of wound healing in rat skin following incision with a novel picosecond infrared laser (PIRL) and different surgical modalities. Lasers Surg Med 2016;48(4):385-391.

11. Kwiatkowski M, Wurlitzer M, Omidi M, et al. Ultrafast extraction of proteins from tissues using desorption by impulsive vibrational excitation. Angew Chem Int Ed Engl 2015;54(1):285-288.

12. Kwiatkowski M, Wurlitzer M, Krutilin A, et al. Homogenization of human tissues via picosecond-infrared laser (PIRL) ablation: Giving a closer view on the in-vivo composition of protein species as compared to mechanical homogenization. J Proteomics 2016;134: 193-202.

13. Petersen H, Gliese A, Stober Y, et al. Picosecond infrared laser (PIRL) application in stapes surgery-first experience in human temporal bones. Otol Neurotol 2018;39(4):e224-e230.

14. Woolman M, Gribble A, Bluemke E, et al. Optimized mass spectrometry analysis workflow with polarimetric guidance for ex vivo and in situ sampling of biological tissues. Sci Rep 2017;7(1):468.

15. Bascoul-Colombo C, Guschina IA, Maskrey BH, Good M, O’Donnell VB, Harwood JL. Dietary DHA supplementation causes selective changes in phospholipids from different brain regions in both wild type mice and the Tg2576 mouse model of Alzheimer's disease. Biochim Biophys Acta 2016;1861(6):524-537.

16. Welsch U, Kummer W, Deller T. Histologie-Das Lehrbuch. 5th ed. Urban \& Fischer Verlag/Elsevier GmbH. 2018 445451. 650-651, 653-658, 660-661

17. Jarmusch AK, Alfaro CM, Pirro V, Hattab EM, Cohen-Gadol AA, Cooks RG. Differential lipid profiles of normal human brain matter and gliomas by positive and negative mode desorption electrospray ionization-mass spectrometry imaging. PLOS One 2016;11(9):e0163180.

18. Lacroix M, Abi-Said D, Fourney DR, et al. A multivariate analysis of 416 patients with glioblastoma multiforme: prognosis, extent of resection, and survival. J Neurosurg 2001;95(2):190-198.

19. Robert-Koch-Institut. Berlin. Gesellschaft der epidemiologischen Krebsregister in Deutschland e.V.: Krebs in Deutschland 2011/2012. 2015;10.

20. Senft C, Bink A, Franz K, Vatter H, Gasser T, Seifert V. Intraoperative MRI guidance and extent of resection in glioma surgery: a randomised, controlled trial. Lancet Oncol 2011;12(11):997-1003.

21. Eljamel MS, Mahboob SO. The effectiveness and costeffectiveness of intraoperative imaging in high-grade glioma resection; a comparative review of intraoperative ALA, fluorescein, ultrasound and MRI. Photodiagnosis Photodyn Ther. 2016;16: 35-43.

22. Barone DG, Lawrie TA, Hart MG. Image guided surgery for the resection of brain tumours. Cochrane Database Syst Rev 2014;(1):CD009685.

23. Stummer W, Pichlmeier U, Meinel T, et al. Fluorescenceguided surgery with 5-aminolevulinic acid for resection of malignant glioma: a randomised controlled multicentre phase III trial. Lancet Oncol 2006;7(5):392-401.

24. Coburger J, Wirtz CR. Fluorescence guided surgery by 5-ALA and intraoperative MRI in high grade glioma: a systematic review. JNO 2019;141(3):533-546.

25. Eberlin LS, Norton I, Dill AL, et al. Classifying human brain tumors by lipid imaging with mass spectrometry. Cancer Res 2012;72(3):645-654.

26. Woolman M, Tata A, Bluemke E, Dara D, Ginsberg HJ, Zarrine-Afsar A. An assessment of the utility of tissue smears in rapid cancer profiling with desorption electrospray ionization mass spectrometry (DESI-MS). J Am Soc Mass Spectrom 2017;28(1):145-153. 